\title{
Medically unexplained physical symptoms in children in a non-specialist paediatric setting
}

\author{
H Perera ${ }^{1}$, ASG Asiri ${ }^{2}$ \\ Sri Lanka Journal of Child Health, 2007; 36: 102-106
}

(Key words: somatization, medically unexplained physical symptoms, recurrent abdominal pain, functional symptoms)

\begin{abstract}
Introduction Medically unexplained symptoms (MUS) are equally common and disabling across countries and cultures. Most prevalent symptoms identified in children are recurrent abdominal pain (RAP), headache, limb pain, and fatigue.
\end{abstract}

Objectives To identify the presenting profile of MUS and associated features in a hospital based, nonspecialist paediatric outpatient setting.

Method Randomly selected children attending the study setting were screened to identify those who fit a working definition for MUS, which specified undiagnosed symptoms being present for 3 months or more. Parents of the identified children answered an interviewer administered semi-structured questionnaire designed to investigate the profile of symptoms, their impact on the child's life, beliefs about the cause and anticipated prognosis and any associated stressful experiences in the child's life.

Results 125 children (mean age 7.8 years) were identified with MUS. Commonest presenting symptoms were abdominal pain and headache. On average, 6.01 medical consultations were made for the symptoms. Difficulties with attending school and studying were the most prominent impact of the symptoms. Majority of the mothers believed that an underlying physical disorder was present, but an associated psychologically stressful experience was identified by them in $51.2 \%$ of cases.

Conclusion Children with MUS warrant extended assessments for associated psychosocial and behavioural factors to enable effective management.

${ }^{1}$ Professor, ${ }^{2}$ Demonstrator, Department of Psychological Medicine, University of Colombo.

(Received on 3 August 2006)

\section{Introduction}

In many patients who present with pain, weakness or fatigue, the symptoms remain unexplained as identifiable disease, even after extensive assessment ${ }^{1}$. General terms used to refer such symptoms and clinical states include "somatization", "somatoform", "medically unexplained symptoms", "abnormal illness behaviour" and "functional symptoms", Such patients are also defined as those with a history of repeated visits to the doctor but "found nothing wrong" . In a World Health Organization (WHO) study, such symptoms were found to be equally common and disabling across countries and cultures ${ }^{4}$. In this paper, the term "medically unexplained symptoms" (MUS) will be used to incorporate all the above mentioned terminology. In general population surveys of children, parents have reported frequent distressing somatic symptoms of different nature ${ }^{5}$. For example, $50 \%$ of 3-12 year olds complained of at least one symptom in the previous two weeks, with the prevalence of recurrent abdominal pain (RAP), headache, limb pains and fatigue being $25-30 \%{ }^{6}$. RAP alone was $10-25 \%$ in school age children ${ }^{7,8}$. On follow up of children with RAP for a minimum of 5 years, it has been found that over $90 \%$ turn out to be of functional nature ${ }^{9,10}$. Other population surveys showed that $2-10 \%$ of children have "functional aches and pains" without an identifiable cause ${ }^{11}$. Furthermore, presentation with pseudo-neurological signs has been reported as common in Asian cultures $^{12}$. Available evidence suggests that biological, psychological, social and interpersonal phenomena are important as precipitating, predisposing and perpetuating factors for MUS ${ }^{1,13}$. In addition, clinical presentations can be complicated by iatrogenic influences from medical interventions or ambiguous and contradictory explanations given for the symptoms ${ }^{1,9,14}$. It is also noteworthy that somatic complaints were the commonest type of clinical presentation seen in children with mental health problems, where this is true of even up to $95 \%$ to $98 \%$ of cases $^{15,16}$. 


\section{Objectives}

The aim of this study was to identify and describe the profile of clinical presentations of MUS, beliefs and concerns related to such symptoms in the parents and associated life experiences in the child. We did not intend to identify the psychiatric morbidity or diagnoses in the subjects concerned. The specific objectives were as follows.

- $\quad$ To identify the symptom profiles in children who presented to a hospital based non specialist paediatric outpatient setting with MUS.

- To identify the impact of MUS on the child's life and emotional state.

- To identify the parental beliefs about the cause and the anticipated outcome of the child's symptoms.

\section{Method}

For this study, the working definition for MUS in children was "recurrent or persistent symptom/s for three months or more, where despite repeated assessment and investigation by the doctor, a cause for the symptom/s was not identified, or a satisfactory outcome from treatment was has not been achieved".

Children attending the outpatient department (OPD) at the Lady Ridgeway Hospital (LRH) in 2005 were randomly selected for screening for those who fulfilled the working definition for MUS. A total of ten separate visits were made to the study setting, over a period of 4-5 months, to identify the study sample and gather the data, so that a reasonable cross section of the population was obtained. Following the identification of the children who fulfilled the working definition for MUS, a semi-structured questionnaire was administered by a trained interviewer to the parent who accompanied the child. Informed voluntary consent was obtained from all respondents to the questionnaire.

The knowledge and experience available from assessments of children with MUS in a specialist child mental health service was useful in designing the semi-structured questionnaire in a culturally relevant format. The questionnaire focused on identifying the following information. (i) Patient demography; (ii) Type and duration of symptom/s; (iii) The number of visits made to see the doctor for the symptom/s; (iv) Impact of the symptoms on the child's daily activities and emotional state; (v) Parental beliefs about the cause of the symptom/s; (vi) Parental beliefs about the possible prognosis of the symptom/s; (vii) Whether the parent was aware of any prevailing fears, worries and distressing experiences in the child's life and if so, the offending source. The questionnaire was pre-tested on both clinical and non-clinical children with MUS and was found to be having a satisfactory test-retest and interrater reliabilities.

\section{Results}

One out of 7 to 10 children $(14.3 \%$ to $10 \%)$ of the initial randomly selected large groups obtained on ten visits to the study setting fit the working definition for MUS. A study sample of 125 children was obtained from this screening. Ages of the children ranged from 2 to 12 years (mean age 7.8 years, SD 2.4, mode 8 years, median 8 years). $61.6 \%$ were male. The duration of the presenting symptom/s ranged from 3 months to 126 months (mean 8.9 months, SD 5.3). The number of presenting physical symptoms ranged from 1 to 4 , and their frequency distribution is given in table 1.

Table 1

Frequency distribution of the number of presenting somatic symptoms

\begin{tabular}{|l|c|}
\hline No. of symptoms & n (\%) \\
\hline One symptom only & $95(76.0)$ \\
\hline Two symptoms & $21(16.8)$ \\
\hline Three symptoms & $07(05.6)$ \\
\hline Four symptoms & $02(01.6)$ \\
\hline
\end{tabular}

The frequency distribution of the types of presenting somatic symptoms is given in table 2 .

Table 2

Frequency distribution of the types of presenting somatic symptoms

\begin{tabular}{|l|l|}
\hline Symptoms & $\begin{array}{l}\mathbf{N} \text { \% } \\
\mathbf{n = 1 2 5}\end{array}$ \\
\hline Headache & $56(44.8)$ \\
\hline Abdominal pain & $56(44.8)$ \\
\hline Body aches & $16(12.8)$ \\
\hline Loss of consciousness & $07(05.6)$ \\
\hline Joint pain & $07(05.6)$ \\
\hline $\begin{array}{l}\text { Other symptoms (chest pain, } \\
\text { dizziness lethargy, blurred vision) }\end{array}$ & $22(17.6)$ \\
\hline
\end{tabular}

Headache and abdominal pain were the commonest symptoms and complained $89.6 \%$ of children. Age distribution in the presentation of these two symptoms is given in table 3 . 
Table 3

Frequency distribution of headache and abdominal pain according to age

\begin{tabular}{|l|l|l|}
\hline Age & $\begin{array}{l}\text { Headache } \\
(n=125)\end{array}$ & $\begin{array}{l}\text { Abdominal pain } \\
(n=125)\end{array}$ \\
\hline 2-5 years & $08(04.5 \%)$ & $12(06.7 \%)$ \\
\hline 6-9 years & $32(17.9 \%)$ & $37(20.7 \%)$ \\
\hline $10-12$ years & $16(09.0 \%)$ & $07(03.6 \%)$ \\
\hline Total & $56(44.8 \%)$ & $56(44.8 \%)$ \\
\hline
\end{tabular}

Mean number of medical consultations made for the presenting symptom/s was 6 (range 1 to 36: SD 5.6).

\section{Impact of the symptoms}

The frequency distribution of impact of symptoms on the child's daily activities and emotional state, as identified by the parent is given in Table 4 .

Table 4

Frequency of impact of symptoms on child's daily activities \& emotional state, as identified by parent

\begin{tabular}{|l|l|}
\hline Impact of symptoms & n (\%) \\
\hline Could not attend school & $72(57.6)$ \\
\hline Was unable to study & $57(45.6)$ \\
\hline Was unable to play & $51(40.8)$ \\
\hline Was unable to travel & $18(14.4)$ \\
\hline Became very distressed with symptom & $55(44.0)$ \\
\hline $\begin{array}{l}\text { Other effects (reduced eating, disturbed } \\
\text { sleep, restlessness, irritability) }\end{array}$ & $41(32.8)$ \\
\hline
\end{tabular}

\section{Attributed causes of the symptoms}

In the opinion of the parents, the possible causes for the symptoms fell into a wide range. Nearly one third did not identify a cause. The frequency distribution of cause of the symptoms, as identified by the parent, is given in table 5 .

Table 5

Frequency distribution of cause of the symptoms as identified by the parent

\begin{tabular}{|l|l|}
\hline Cause of symptoms - parent opinion & $\mathrm{N}(\%)$ \\
\hline Result of a previous injury / trauma & $20(16.0)$ \\
\hline $\begin{array}{l}\text { Cause within the abdomen - (worms, } \\
\text { gastritis, food allergy) }\end{array}$ & $21(16.8)$ \\
\hline Infection & $08(06.4)$ \\
\hline Psychological & $05(04.0)$ \\
\hline Don't know / unable to guess & $30(24.0)$ \\
\hline Not definite / some physical problem & $08(06.4)$ \\
\hline Tumour & $04(03.2)$ \\
\hline Epilepsy & $01(0.8)$ \\
\hline Arthritis & $03(02.4)$ \\
\hline Other & $25(31.3)$ \\
\hline Total & $125(100)$ \\
\hline
\end{tabular}

\section{Anticipated prognosis}

In response to the inquiry about anticipated prognosis, in $50(40 \%)$ cases parents were worried whether symptoms will progress into a serious or even a life threatening illness. In 33 (29.6\%), deterioration in schoolwork was anticipated. In 4 (3.2\%) an adverse effect on the growth of the child and in another $4(3.2 \%)$ difficulty with walking was expected. In $3(2.4 \%)$, a spontaneous resolution of symptoms was considered and in $6(4.8 \%)$ a miscellany of other outcomes were expected. All other $25(20 \%)$ responded with "don't know".

\section{Identified stressful experiences}

The parents offered the following responses when asked whether they were aware of any prevailing fears, worries and distressing experiences in the child's life at present and if so, the offending source. In $42(33.6 \%)$ cases, the parent did not identify such an experience. In $19(15.2 \%)$, the response was "don't know". In 64 (51.2\%), the parent identified a possible source of stress in the child. The frequency distribution of sources of fear / anxiety / stress as identified by the parent is given in table 6 .

Table 6

Frequency distribution of sources of fear /anxiety / stress as identified by the parent

\begin{tabular}{|l|l|l|}
\hline $\begin{array}{l}\text { Source of fear/ anxiety } \\
\text { stress }\end{array}$ & $\mathbf{n}(\mathbf{\% )}$ & $\begin{array}{l}\text { Cumulative } \\
\text { \% }\end{array}$ \\
\hline $\begin{array}{l}\text { Fear of separation from } \\
\text { parent }\end{array}$ & $16(12.8)$ & $16(12.8)$ \\
\hline $\begin{array}{l}\text { Difficulty in adjustment } \\
\text { to new school / class }\end{array}$ & $11(08.8)$ & $27(21.6)$ \\
\hline $\begin{array}{l}\text { Relationship difficulty } \\
\text { with class teacher }\end{array}$ & $10(08.0)$ & $37(29.6)$ \\
\hline Rivalry with a sibling & $09(07.2)$ & $46(36.8)$ \\
\hline $\begin{array}{l}\text { Stress in home } \\
\text { environment }\end{array}$ & $09(07.2)$ & $55(44.0)$ \\
\hline $\begin{array}{l}\text { Stress related to } \\
\text { neighbourhood } \\
\text { environment }\end{array}$ & $04(03.2)$ & $59(47.2)$ \\
\hline $\begin{array}{l}\text { Illness in a family } \\
\text { member }\end{array}$ & $04(03.2)$ & $63(50.4)$ \\
\hline $\begin{array}{l}\text { Relationship difficulty } \\
\text { with peers }\end{array}$ & $01(0.8)$ & $64(51.2)$ \\
\hline No stressful source & $42(33.6)$ & $106(84.8)$ \\
\hline $\begin{array}{l}\text { Unable to give an } \\
\text { answer - “don't know" }\end{array}$ & $19(15.2)$ & $19(15.2)$ \\
\hline
\end{tabular}




\section{Discussion}

The working definition of the study limited the inquiry to those children with MUS of 3 months or more. Hence, the children with MUS of lesser duration did not get included in the sample. Nevertheless, $10 \%$ to $14 \%$ of the population attending the study setting fulfilled the criteria of MUS. It is also noteworthy that for an average duration 8.9 months, the symptoms continued unabated and undiagnosed. In the WHO study that investigated 14 countries, the prevalence MUS was found to be $19.7 \%$ on average and $19.6 \%$ in India ${ }^{4}$. With regard to clinical presentation, multiple symptoms are reported to be common in community samples $^{5,7}$. In contrast, over $75 \%$ of children in our hospital-based study complained of only one symptom even though the assessment tool provided for exploring the presence of multiple symptoms. The literature did not provide any studies that had specifically addressed single symptom presentation in MUS. However, two specific symptoms namely, abdominal pain or headache are the most common and widely recognized presentations of $\mathrm{MUS}^{7,8,17}$. Our study replicates this finding with $89.2 \%$ complaining of either abdominal pain or headache. In contrast to most studies that found RAP to be more prevalent, abdominal pain and headache were in equal proportions in ours (Table 2). With regard to age distribution of the symptoms, it is known that abdominal pain is commoner around 9 years and headache around 3 years later ${ }^{17}$. This finding is replicated by our study too (Table 3 ). Regarding the gender distribution, we had a higher prevalence of boys in our sample, though a gender difference has not been found with MUS $^{7,13,18}$. The difference in sample type may explain this gender bias as most reported studies are community based rather than hospital based.

Another relevant observation in our study was that an average of 6 consultations and as many as 36 were made for the same somatic complaint/s. This finding is consistent with the high health service utilization seen among children with MUS ${ }^{3,8,19}$. This observation is explained on the basis of several child and parent related factors associated with MUS, such as the affected children having more emotional and behavioural difficulties and being perceived as "sickly", anxious, sensitive to pain and having adjustment difficulties ${ }^{3,18,20,21}$. Parents are likely to seek medical help more often for such children. In our sample too, marked distress about the symptoms was reported by parents in $44 \%$ of children. In addition, expecting the symptoms to become serious or life threatening $(40 \%)$ and anticipation of deterioration of school work (29.6\%) may have reinforced parental worries into seeking medical help repeatedly. Parental anxiety is recognized to play an important role in MUS where they perceive the children to have a low quality of life and a lesser functioning level than that measured objectively ${ }^{22,23}$.

Though symptoms presented without any evidence of a physical disorder for an average of 8.9 months, a clear impact on several aspects of the child's functioning and the emotional state was identified in our study (table 4). MUS presenting as RAP and headache are recognized to be associate with school absenteeism in both school age and preschool age children $^{18,24,25}$. Also, a high prevalence of academic difficulties has been recognized among children with MUS ${ }^{3,5,25}$. It is possible to postulate that at least 30\% of children in our sample had similar problems though not directly studied, as $57.6 \%$ missed school and $45.6 \%$ could not study, but play and travel was not affected to the same extent (Table 4). Also, the stresses and fears in children reported by parents were mainly related to school setting and deterioration in schoolwork was anticipated in nearly $30 \%$ (table 6).

Although a psychological cause for the symptoms was considered in only $4 \%$ of cases by the parents, a current stressful life experience was identified subsequently in $51.2 \%$ of children (tables $5 \& 6$ ). It is known that experience of stress may act as a precipitating, predisposing or perpetuating factor in the aetiology of $\mathrm{MUS}^{3,13,26}$. Our investigation was limited to eliciting the presence or absence of stressful experiences but $33 \%$ to $50 \%$ of children with MUS are thought to have psychiatric comorbidity, especially anxiety and depression ${ }^{7,13,19,27}$. A further observation that deserves comment is that nearly $25 \%$ of parents did not suggest a possible cause for the child's symptoms or the anticipated prognosis. A possible reason is firstly, the culture of passivity shown by the Sri Lankan public towards medical authority. Secondly, the general public may associate somatic complaints to be always associated with physical disorders. Thirdly, stigma may prevent acknowledgement of a psychological cause for the symptoms.

\section{Clinical relevance}

This study shows that children with MUS warrant further extended assessment beyond their presenting physical symptom. This is necessary in order to identify any associated psychological and environmental factors to enable satisfactory management. The questionnaire used in this study 
could be a useful tool in screening such children in general paediatric settings.

\section{Acknowledgements}

The contribution made by the medical students of the University of Colombo in gathering the data and the cooperation given by the medical officers attached to the outpatient department of Lady Ridgeway Hospital, is greatly appreciated.

\section{References}

1. Mayou R, Farmer A. Functional somatic symptoms and syndromes. British Medical Journal 2002; 325: 265-8.

2. Lipowzki ZJ. Somatization: the concept and its clinical application. American Journal of Psychiatry 1988; 145: 1358-68.

3. Campo JV, Janssen-McWilliams L, Comer DM, Kelleher KJ. Somatization in paediatric primary care: association with psychopathology, functional impairment, and use of services. Journal of the American Academy of Child and Adolescent Psychiatry 1999; 38: 1093-101.

4. Gureje O, Simon GE, Ustun TB, Goldberg DP. Somatization in cross-cultural perspective: a World Health Organization study in primary care. American Journal of Psychiatry 1997; 154: 989-95.

5. Garralda ME. Assessment and management of somatisation in childhood and adolescence: a practical perspective. Journal of Child Psychology and Psychiatry 1999; 40: 1159-67.

6. Kramer T, Garralda ME. Child and adolescent mental health problems in primary care. Advances in Psychiatric Treatment 2000; 6: 287 94.

7. Garber J, Walker LS, Zeman J. Somatization symptoms in a community sample of children and adolescents: further validation of Children's Somatization Inventory, psychological assessment. Journal of Consulting and Clinical Psychology 1991; 3: 588-95.
8. Offord DR, Boyle MH, Szatmari P, Rae-Grant NL, Links PS, Cadman DT, Byles JA, Crawford JW, Munrow BH, Byrne C, Thomas H, Woodward CA. Ontario Child Health Study II. Six month prevalence of disorder and rates of service utilization. Archives of General Psychiatry 1987; 44: 833-6.

9. Stickler GB, Murphy DB. Recurrent abdominal pain. American Journal of Diseases of Children 1979; 133: 486-9.

10. Bain HW. Chronic vague abdominal pain in childhood. Pediatric Clinics of North America 1974; 21: 91-100.

11. Goodman JE, McGrath PJ. The epidemiology of pain in children and adolescents: a review. Pain 1991; 46: 247-64.

12. Srinath S, Bharat S, Girimaji S, Seshadri S. Characteristics of a child inpatient population with hysteria in India. Journal of the American Academy of Child and Adolescent Psychiatry. 1993; 32: 822-5.

13. Lask B, Fosson A. Childhood illness: the psychosomatic approach. Wiley, Chichester, 1989.

14. Walker LS, Garber J, Greene JW. Somatization symptoms in paediatric abdominal pain patients: relation to chronicity of abdominal pain and parent somatization. Journal of Abnormal Child Psychology 1991; 19: 379-94.

15. Campo JV, Fritsch SL. Somatization in children and adolescents. Journal of the American Academy of Child and Adolescent Psychiatry 1994; 33: 1223-5.

16. Goldberg RJ, Novack DH, Gask L. The recognition and management of somatization: what is needed in primary care training. Psychosomatics 1992; 33: 55-61.

17. Oster J. Recurrent abdominal pain, headache and limb pains in children and adolescents. Pediatrics 1972; 50: 429-36.

18. Zuckerman B, Stevenson J, Bailey V. Stomachaches and headaches in a community sample of preschool children. Pediatrics 1987; 79: $677-82$. 
19. Garralda ME, Bailey D. Children with psychiatric disorders in primary care. Journal of Child Psychology and Psychiatry 1986; 27: 61124.

20. Lask B. The high achieving child. Postgraduate Medical Journal 1986; 62: 143-5.

21. Aromaa M, Sillanpaa M, Rautava P, Helenius H. Pain experience of children with headache and their families: a controlled study. Pediatrics 200; 106: 270-5.

22. Hunfeld JAM, Perquin CW, HazebroekKampschreur AAJM, Passchier J, van Suijlekom-Smit LWA, van der Wouden J. Physically unexplained chronic pain and its impact on children and their families: the mother's perception. Psychology and Psychotherapy: Theory, Research and Practice 2002; 75: 251-60.

23. Youssef NN, Murphy TG, Langsder AL, Rosh JR. Quality of life for children with functional abdominal pain: a comparison study of patients' and parents' perception. Pediatrics 2006; 117: 54-9.
24. Apley J, Naish N. Recurrent abdominal pain: a field survey of 11,000 school children. Archives of Diseases of Childhood 1958; 33: 1656-70.

25. Collins C, Hockaday JM, Waters WE. Headache and school absence. Archives of Disease of Childhood 1985; 60: 245-7.

26. Walker LS, Garber J, Greene JW. Somatic complaints in paediatric patients: a prospective study of the role of negative life events, child social and academic competence and parental somatic symptoms. Journal of Consulting and Clinical Psychology 1994; 62: 1213-21.

27. Konijnenberg AY, de Graeff-Meeder ER, van der Hoeven J, Kimpen JL, Buitelaar JK, Uiterwaal CS. Psychiatric morbidity in children with medically unexplained chronic pain: diagnosis from the pediatrician's perspective. Pediatrics 2006; 117: 889-97. 